\title{
Study of $Z$ Bosons Produced in Association with Charm in the Forward Region
}

\author{
R. Aaij et al. \\ (LHCb Collaboration)
}

(Received 17 September 2021; revised 17 December 2021; accepted 28 January 2022; published 24 February 2022)

\begin{abstract}
Events containing a $Z$ boson and a charm jet are studied for the first time in the forward region of protonproton collisions. The data sample used corresponds to an integrated luminosity of $6 \mathrm{fb}^{-1}$ collected at a center-of-mass energy of $13 \mathrm{TeV}$ with the $\mathrm{LHCb}$ detector. In events with a $Z$ boson and a jet, the fraction of charm jets is determined in intervals of $Z$-boson rapidity in the range $2.0<y(Z)<4.5$. A sizable enhancement is observed in the forwardmost $y(Z)$ interval, which could be indicative of a valencelike intrinsic-charm component in the proton wave function.
\end{abstract}

DOI: 10.1103/PhysRevLett.128.082001

The possibility that the proton wave function may contain a $|u u d c \bar{c}\rangle$ component, referred to as intrinsic charm (IC), in addition to the charm content that arises due to perturbative gluon radiation, i.e., $g \rightarrow c \bar{c}$ splitting, has been debated for decades (for a recent review, see Ref. [1]). The light front QCD calculations of Refs. [2,3], referred to as the BrodskyHoyer-Peterson-Sakai (BHPS) model, predict that nonperturbative IC manifests as valencelike charm content in the parton distribution functions (PDFs) of the proton; whereas, if the $c$-quark content is entirely perturbative in nature, the charm PDF resembles that of the gluon and sharply decreases at large momentum fractions $x$. (Charge conjugation is implied throughout this Letter, e.g., charm refers to both the $c$ and $\bar{c}$ quarks.) Understanding the role that nonperturbative dynamics play inside the nucleon is a fundamental goal of nuclear physics [4-15]. Furthermore, the existence of IC would have many phenomenological consequences. For example, IC would alter both the rate and kinematics of $c$ hadrons produced by cosmic-ray proton interactions in the atmosphere, which are an important source of background in studies of astrophysical neutrinos [16-21]. The cross sections of many processes at the LHC and other accelerators would also be affected [22-32].

Measurements of $c$-hadron production in deep inelastic scattering [33] and in fixed-target experiments [34], where the typical momentum transfers were $Q \lesssim 10 \mathrm{GeV}$ (natural units are used throughout this Letter), have been interpreted both as evidence for [35,36] and against [37] the percentlevel IC content predicted by BHPS. Even though such

\footnotetext{
*Full author list given at the end of the article.

Published by the American Physical Society under the terms of the Creative Commons Attribution 4.0 International license. Further distribution of this work must maintain attribution to the author(s) and the published article's title, journal citation, and DOI. Funded by SCOAP.
}

experiments are in principle sensitive to valencelike $c$-quark content, interpreting these low- $Q$ data is challenging since it requires careful theoretical treatment of nonperturbative hadronic and nuclear effects. Recent global PDF analyses, which also include measurements from the LHC, are inconclusive and can only exclude IC carrying more than a few percent of the momentum of the proton $[38,39]$.

Reference [29] proposed probing IC by studying events containing a $Z$ boson and a charm jet $Z c$ in the forward region of proton-proton $(p p)$ collisions at the LHC. The ratio of production cross sections $\mathcal{R}_{j}^{c} \equiv \sigma(Z c) / \sigma(Z j)$, where $Z j$ refers to events containing a $Z$ boson and any type of jet, was chosen because it is less sensitive than $\sigma(Z c)$ to experimental and theoretical uncertainties. Since $Z c$ production is inherently at large $Q$, above the electroweak scale, hadronic effects are small. A leading-order $Z c$ production mechanism is $g c \rightarrow Z c$ scattering (see Fig. 1), where in the forward region one of the initial partons must have large $x$, hence $Z c$ production probes the valencelike region (Fig. S4 of Supplemental Material shows the $x$ regions probed). Using next-to-leading-order (NLO) standard model (SM) calculations, Fig. 2 illustrates that a percent-level valencelike IC contribution would produce a clear enhancement in $\mathcal{R}_{j}^{c}$ for large (more forward) values of $Z$ rapidity, $y(Z)$; whereas only small effects are expected in the central region where all previous measurements of $\mathcal{R}_{j}^{c}$ were made $[40,41]$.
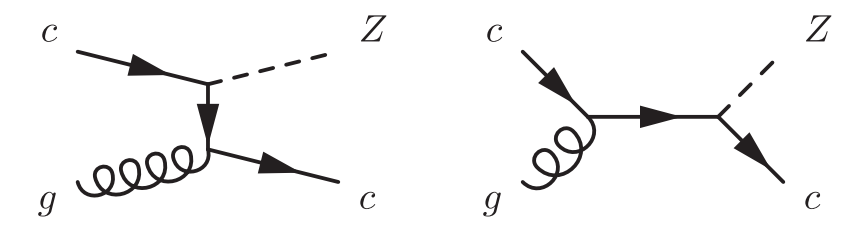

FIG. 1. Leading-order Feynman diagrams for $g c \rightarrow Z c$ production. 


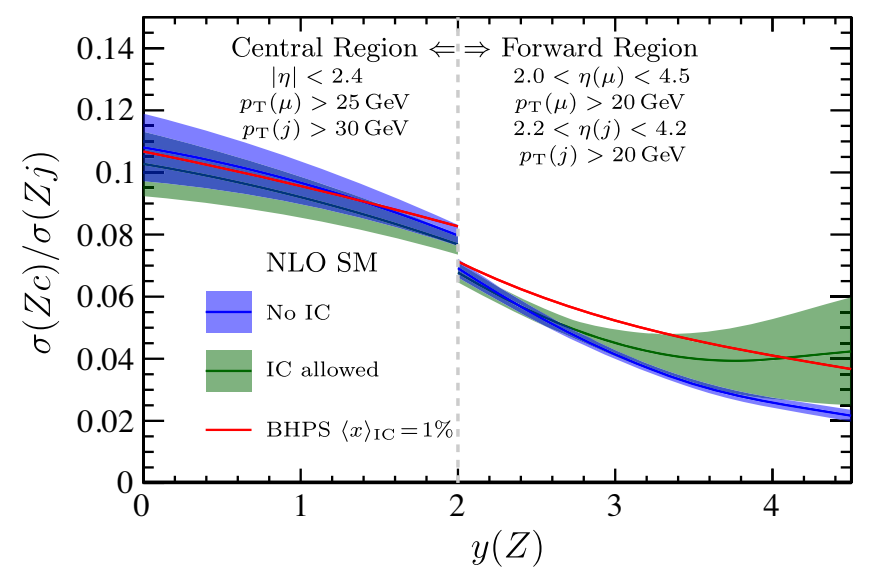

FIG. 2. NLO SM predictions [29] for $\mathcal{R}_{j}^{c}$ without IC [42], allowing for potential IC [39], and with the valencelike IC predicted by BHPS with a mean momentum fraction of $1 \%$ [38]. The fiducial region from Ref. [41] is used for $y(Z)<2$; otherwise the fiducial region of this analysis is employed. The broadening of the error band that arises in the forward region, when allowing for IC, is due to the lack of sensitivity to valencelike IC from previous experiments. More details on these calculations are provided in Supplemental Material [43]. The error bands shown for the first two predictions display the $68 \%$ confidence-level regions. Only the central value is shown for BHPS due to the charm PDF being fixed.

This Letter presents the first measurement of $\mathcal{R}_{j}^{c}$ in the forward region of $p p$ collisions. The data sample used corresponds to an integrated luminosity of $6 \mathrm{fb}^{-1}$ collected at a center-of-mass energy of $\sqrt{s}=13 \mathrm{TeV}$ with the LHCb detector. The $Z$ bosons are reconstructed using the $Z \rightarrow \mu^{+} \mu^{-}$decay, where henceforth all $Z / \gamma^{*} \rightarrow \mu^{+} \mu^{-}$ production in the mass range $60<m\left(\mu^{+} \mu^{-}\right)<120 \mathrm{GeV}$ is labeled $Z \rightarrow \mu^{+} \mu^{-}$. The analysis is performed using jets clustered with the anti- $k_{T}$ algorithm [44] using a distance parameter $R=0.5$. The fiducial region is defined in terms of the transverse momentum $p_{T}$, pseudorapidity $\eta$, and azimuthal angle $\phi$ of the muon and jet momenta, and includes a requirement on $\Delta R(\mu, j) \equiv$ $\sqrt{\Delta \eta(\mu, j)^{2}+\Delta \phi(\mu, j)^{2}}$ to ensure that the muons and jets are well separated, which suppresses backgrounds from QCD multijet events and electroweak processes like $W+$ jet production. Charm jets are the subset for which there is a promptly produced and weakly decaying $c$ hadron within the jet. The fiducial region is defined in Table I. If multiple jets satisfy these criteria, the one with the highest $p_{T}$ is selected. No requirement is placed on the maximum number of jets in the event.

The quantity $\mathcal{R}_{j}^{c}$ is measured in intervals of $y(Z)$ as $\mathcal{R}_{j}^{c}=N(c$-tag $) /[\varepsilon(c-\operatorname{tag}) N(j)]$, where $N(c$-tag $)$ is the observed $Z c$ yield, $\varepsilon(c$-tag $)$ is the $c$-tagging efficiency, and $N(j)$ is the total $Z j$ yield. The integrated luminosity does not enter this expression because $\mathcal{R}_{j}^{c}$ involves a ratio of production cross sections. In addition, the muon and jet reconstruction efficiencies largely cancel in the ratio due to the similarity of the $Z$ boson and jet kinematics in $Z c$ and $Z j$ production. The $c$-tagging algorithm, which is described in detail in Ref. [45], looks for a displaced-vertex (DV) signature inside the jet cone that is indicative of the weak decay of a $c$ hadron.

The $\mathrm{LHCb}$ detector is a single-arm forward spectrometer covering the pseudorapidity range $2<\eta<5$, described in detail in Refs. [46,47]. Simulated data samples are used to evaluate the detector response for jet reconstruction, including the $c$-tagging efficiency, and to validate the analysis. In the simulation, $p p$ collisions are generated using PYTHIA [48] with a specific LHCb configuration [49]. Decays of unstable particles are described by EVTGEN [50], in which final-state QED radiation is generated using PHOTOS [51]. The interaction of the generated particles with the detector, and its response, are implemented using the GEANT4 toolkit [52] as described in Ref. [53].

The online event selection is performed by a trigger $[54,55]$ consisting of a hardware stage using information from the calorimeter and muon systems, followed by a software stage that performs a full event reconstruction. At the hardware stage, events are required to have a muon with $p_{T}(\mu)>6 \mathrm{GeV}$. In the software stage, the muon track is required to be of good quality and to have $p_{T}(\mu)>10 \mathrm{GeV}$. The off-line selection builds $Z \rightarrow \mu^{+} \mu^{-}$candidates from two oppositely charged muon tracks that must be in the fiducial region defined in Table I and consistent with originating directly from the same $p p$ collision.

Jet reconstruction is performed off-line by clustering charged and neutral particle-flow candidates [56] using the anti- $k_{T}$ clustering algorithm as implemented in FASTJET [57]. Reconstructed jets with $15<p_{T}(j)<100 \mathrm{GeV}$ and $2.2<\eta(j)<4.2$ are kept for further analysis. Jets with $15<p_{T}(j)<20 \mathrm{GeV}$, which are outside of the fiducial region, are retained for use when unfolding the detector response. The $\eta(j)$ requirement, which is included in the fiducial region and was first used in Refs. [58-60], ensures a nearly uniform $c$-tagging efficiency of about $24 \%$, with minimal $p_{T}(j)$ or $\eta(j)$ dependence. The fiducial requirement $\Delta R(\mu, j)>0.5$ is applied to reconstructed jets. Finally, the highest- $p_{T}$ jet satisfying these criteria from the same $p p$ collision as the $Z$ boson is selected. After applying all requirements, $68694 \mathrm{Zj}$ candidates remain in the dataset.

The effects of the detector response on the measured jet momenta are accounted for using an unfolding procedure. This involves first determining the reconstructed $Z c$ and $Z j$ yields in intervals of $\left[y(Z), p_{T}(j)\right]$. The non- $Z$ background is neglected for both $Z c$ and $Z j$ candidates because it is less than $1 \%$ and largely cancels in the $\mathcal{R}_{j}^{c}$ ratio. The $c$-jet yields are determined using the DV-based tagging approach described in detail in the following paragraphs. Interval migration is accounted for by unfolding the $p_{T}(j)$ distributions of the $Z c$ and $Z j$ yields in each $y(Z)$ interval 
TABLE I. Definition of the fiducial region.

\begin{tabular}{lc}
\hline \hline$Z$ bosons & $p_{T}(\mu)>20 \mathrm{GeV}, 2.0<\eta(\mu)<4.5$, \\
$60<m\left(\mu^{+} \mu^{-}\right)<120 \mathrm{GeV}$ \\
Jets & $20<p_{T}(j)<100 \mathrm{GeV}, 2.2<\eta(j)<4.2$ \\
Charm jets & $p_{T}(c$ hadron $)>5 \mathrm{GeV}, \Delta R(j, c$ hadron $)<0.5$ \\
Events & $\Delta R(\mu, j)>0.5$ \\
\hline \hline
\end{tabular}

independently using an iterative Bayesian procedure $[61,62]$. The $Z c$ yields are then corrected for $c$-tagging inefficiency. Finally, the unfolded $\left[y(Z), p_{T}(j)\right]$ distributions are integrated for $p_{T}(j)>20 \mathrm{GeV}$ to obtain the $Z c$ and $Z j$ yields used to determine the $\mathcal{R}_{j}^{c}$ ratios. The analysis employs three $y(Z)$ intervals with ranges 2.00-2.75, 2.75-3.50, and 3.50-4.50, and four $p_{T}(j)$ intervals ranging $15-20,20-30,30-50$, and $50-100 \mathrm{GeV}$, where after unfolding the yields in the three highest $p_{T}(j)$ intervals are summed to obtain $\mathcal{R}_{j}^{c}$.

The signature of a $c$ jet is the presence of a long-lived $c$ hadron that carries a sizable fraction of the jet energy. The tagging of $c$ jets is performed using DVs formed from the decays of such $c$ hadrons. The choice of using DVs and not single-track or other non-DV-based jet properties, e.g., the number of particles in the jet, is driven by the need for a small misidentification probability of light-parton jets. Furthermore, the properties of DVs from $c$-hadron decays are known to be well modeled by simulation, which means that only small corrections using control samples are required. Since DVs can also be formed from the decays of $b$ hadrons or due to artifacts of the reconstruction, the DV-tagged charm yields are obtained by fitting the distributions of DV features with good discrimination power between $c, b$, and light-parton jets.

The tracks used as inputs to the DV-tagger algorithm are required to have $p_{T}>0.5 \mathrm{GeV}$ and to be inconsistent with originating directly from a $p p$ interaction point. A DV is associated to a jet when $\Delta R<0.5$ between the jet axis and the DV direction of flight, defined by the vector from the $p p$ interaction point to the DV position. Requirements that reject strange-hadron decays and particles formed in interactions with material [63] are placed on the mass, $m(\mathrm{DV})$, and momentum, $p(\mathrm{DV})$, of the particles that form the DV, along with the DV position. In addition, only DVs with at most four tracks are used, since higher-multiplicity DVs are almost exclusively due to $b$-hadron decays. More details about the $c$-tagging algorithm are provided in Ref. [45].

Two DV properties are used to separate charm jets from beauty and light-parton jets: the number of tracks in the DV, $N_{\text {trk }}(\mathrm{DV})$, and the corrected mass, $m_{\text {cor }}(\mathrm{DV}) \equiv$ $\sqrt{m(\mathrm{DV})^{2}+[p(\mathrm{DV}) \sin \theta]^{2}}+p(\mathrm{DV}) \sin \theta$, where $\theta$ is the angle between the momentum and the flight direction of the DV. The corrected mass, which is the minimum mass the long-lived hadron can have that is consistent with the flight direction, peaks near the typical $c$-hadron mass for $c$ jets, and consequently provides excellent discrimination against other jet types. The DV track multiplicity provides additional discrimination against $b$ jets, since $b$-hadron decays often produce many displaced tracks. These two distributions are fitted simultaneously to obtain the DV-tagged $c$-jet yields. The probability density functions, referred to as templates, for $c, b$, and light-parton jets are obtained from calibration data samples that are each highly enriched in a given jet flavor [45]. Figure 3 shows the $m_{\text {cor }}(\mathrm{DV})$ and $N_{\text {trk }}(\mathrm{DV})$ distributions for all DV-tagged candidates in the $Z j$ data sample reconstructed in the fiducial region, along with the fit projections; such fits are performed in each $\left[y(Z), p_{T}(j)\right]$ interval to obtain the reconstructed $Z c$ yields.

The effects of $p_{T}(j)$ interval migration are corrected for using the unfolding procedure. The detector response is studied using the $p_{T}$-balance distribution $p_{T}(j) / p_{T}(Z)$ for $Z j$ candidates that are nearly back-to-back in the transverse plane, using the same technique as in Refs. [56,64]. Small adjustments are applied to the $p_{T}(j)$ scale and resolution in simulation to obtain the best agreement with data. In addition, for the $Z c$ and $Z j$ samples the $p_{T}(j)$ and $p_{T}(\mathrm{DV})$ distributions in simulation are adjusted to match those observed in data. The unfolding matrix for jets that contain a reconstructed DV is shown in Fig. 4, while the corresponding matrix for inclusive $Z j$ production is provided in Supplemental Material [43].
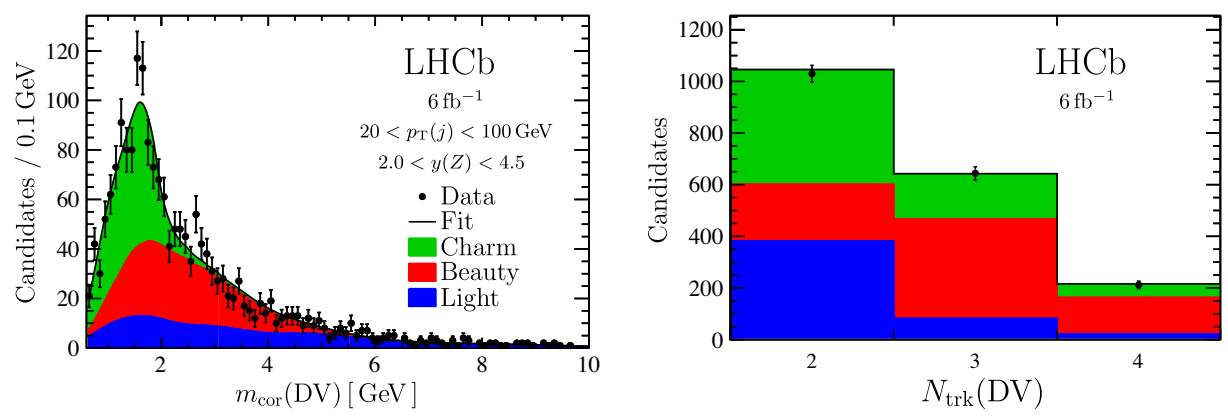

FIG. 3. Distributions of (left) $m_{\text {cor }}(\mathrm{DV})$ and (right) $N_{\text {trk }}(\mathrm{DV})$ for all DV-tagged candidates in the $Z j$ data sample reconstructed in the fiducial region with the projections of the fit results superimposed. 


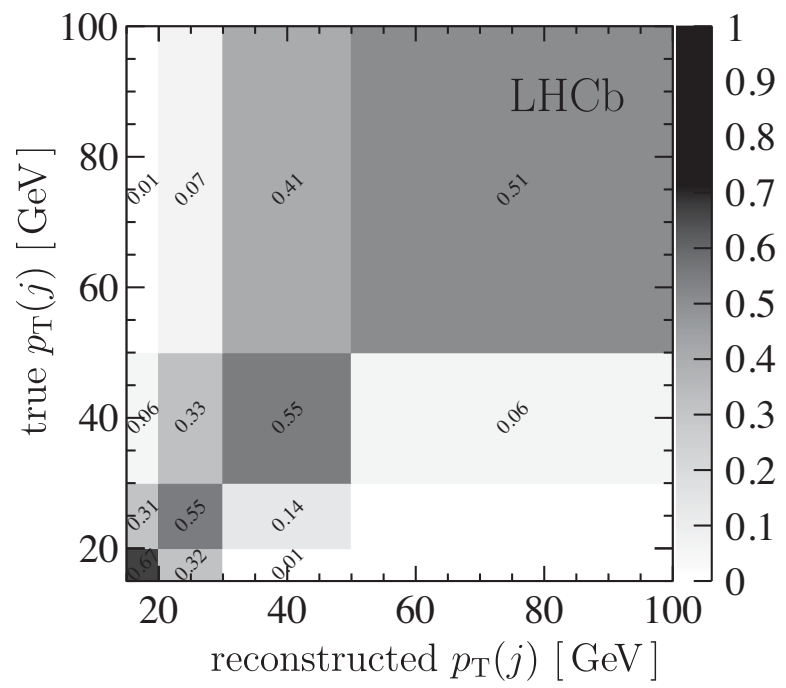

FIG. 4. The detector-response matrix for $c$-tagged jets. The shading represents the interval-to-interval migration probabilities ranging from (white) 0 to (black) 1 . Numerical labels are only shown for values greater than $1 \%$. Jets with true (reconstructed) $p_{T}(j)$ in the 20-100 GeV region but for which the reconstructed (true) $p_{T}(j)$ is either below $15 \mathrm{GeV}$ or above $100 \mathrm{GeV}$ are included in the unfolding but not shown graphically.

The dominant systematic uncertainty is due to limited knowledge of the $c$-tagging efficiency, which is measured in $p_{T}(j)$ intervals using data in Ref. [45] and briefly summarized here. Scale factors that correct for discrepancies between data and simulation are determined using a tag-and-probe approach on a dijet calibration sample. A stringent requirement is applied to the tag jet which enriches the probe-jet sample in charm content. The DVtagged $c$-jet yield in the probe sample is obtained in the same way the $Z c$ yield is determined in this analysis, namely by fitting the $m_{\text {cor }}(\mathrm{DV})$ and $N_{\text {trk }}(\mathrm{DV})$ distributions for DV-tagged probe jets. The total number of $c$ jets in the probe sample is obtained by fully reconstructing the $D^{0} \rightarrow K^{-} \pi^{+}$and $D^{+} \rightarrow K^{-} \pi^{+} \pi^{+}$decays, obtaining the prompt-charm yields by fitting the $D$-meson mass and impact-parameter distributions, then correcting these yields for the detector response, decay branching fractions [65], and $c$-hadron fragmentation fractions [66]. The $c$-tagging efficiency is the ratio of the DV-tagged and total $c$-jet probe-sample yields. The scale factors that correct the $c$-tagging efficiency in simulation are determined to be $1.03 \pm 0.06,1.01 \pm 0.08$, and $1.09 \pm 0.17$ in the $20-30$, $30-50$, and $50-100 \mathrm{GeV} p_{T}(j)$ intervals, respectively, with corresponding $c$-tagging efficiencies of $(23.9 \pm 1.4) \%$, $(24.4 \pm 1.9) \%$, and $(23.6 \pm 4.1) \%$. These uncertainties, which include all statistical and systematic contributions, are propagated to the $\mathcal{R}_{j}^{c}$ results producing 6\%-7\% relative uncertainties in each $y(Z)$ interval.

Other sources of smaller systematic uncertainty are also considered. First, variations of the $m_{\text {cor }}(\mathrm{DV})$ and $N_{\text {trk }}(\mathrm{DV})$
TABLE II. Relative systematic uncertainties on $\mathcal{R}_{j}^{c}$, where ranges indicate that the value depends on the $y(Z)$ intervals.

\begin{tabular}{lc}
\hline \hline Source & Relative uncertainty \\
\hline$c$ tagging & $6 \%-7 \%$ \\
DV-fit templates & $3 \%-4 \%$ \\
Jet reconstruction & $1 \%$ \\
Jet $p_{T}$ scale and resolution & $1 \%$ \\
Total & $8 \%$ \\
\hline \hline
\end{tabular}

templates are studied, which arise from using different strategies to model the backgrounds in the highly enriched calibration data samples. However, the shifts observed in the $Z c$ yields largely cancel with the corresponding shifts seen in $\varepsilon(c$-tag). The residual differences of $3 \%-4 \%$ in each $y(Z)$ interval are assigned as systematic uncertainties. The ratio of the jet-reconstruction efficiency for $c$ and inclusive jets is consistent with unity in all kinematic intervals in simulation, with a $1 \%$ systematic uncertainty assigned due to the limited sample sizes. Finally, the statistical precision of the back-to-back $Z j$ sample used to determine the $p_{T}(j)$ scale and resolution is propagated through the unfolding procedure resulting in a $1 \%$ relative systematic uncertainty on $\mathcal{R}_{j}^{c}$. The systematic uncertainties are summarized in Table II.

Figure 5 shows the measured $\mathcal{R}_{j}^{c}$ distribution in intervals of $y(Z)$; the numerical results are provided in Table III, and additional results are reported in Supplemental Material [43]. The measured $\mathcal{R}_{j}^{c}$ values are compared to NLO SM calculations [29] based on Refs. [67-73], which are validated against additional predictions $[70,71,74,75]$ and updated here to use more recent PDFs [38,39,42,76,77].

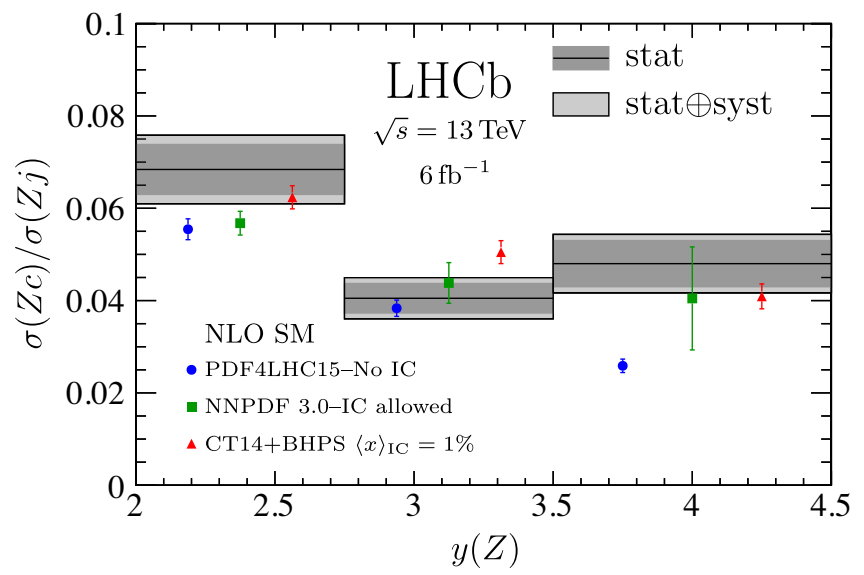

FIG. 5. Measured $\mathcal{R}_{j}^{c}$ distribution (gray bands) for three intervals of forward $Z$ rapidity, compared to NLO SM predictions [29] without IC [42], with the charm PDF shape allowed to vary (hence, permitting IC) [39,76], and with IC as predicted by BHPS with a mean momentum fraction of $1 \%$ [38]. The predictions are offset in each interval to improve visibility. 
TABLE III. Numerical results for the $\mathcal{R}_{j}^{c}$ measurements, where the first uncertainty is statistical and the second is systematic.

\begin{tabular}{lc}
\hline \hline$y(Z)$ & $\mathcal{R}_{j}^{c}(\%)$ \\
\hline $2.00-2.75$ & $6.84 \pm 0.54 \pm 0.51$ \\
$2.75-3.50$ & $4.05 \pm 0.32 \pm 0.31$ \\
$3.50-4.50$ & $4.80 \pm 0.50 \pm 0.39$ \\
$2.00-4.50$ & $4.98 \pm 0.25 \pm 0.35$ \\
\hline \hline
\end{tabular}

While $Z c$ predictions at next-to-next-to-leading-order in QCD are not available, $Z b$ predictions are [78], and similar methods should be applicable. The NNPDF Collaboration analysis provides results where the charm PDF is allowed to vary, both in size and in shape [39]. The sizable uncertainties that arise in the forward region are due to the lack of sensitivity to valencelike IC from previous experiments. Reference [38] updated the CT14 analysis [79] to include the IC content predicted by BHPS [2,3], which results in the enhancement at forward $y(Z)$ shown previously in Fig. 2. These predictions have smaller uncertainties because the size and shape of the IC contribution are fixed, i.e., the IC contribution is assumed to be known, hence does not contribute to the uncertainty on $\mathcal{R}_{j}^{c}$. More details on the theory calculations, along with predictions based on other PDFs [80-82], are provided in Supplemental Material [43].

The observed $\mathcal{R}_{j}^{c}$ values are consistent with both the no-IC and IC hypotheses in the first two $y(Z)$ intervals; however, this is not the case in the forwardmost interval where the ratio of the observed to no-IC-expected values is $1.85 \pm 0.25$. As illustrated in Fig. 2, this is precisely the $y(Z)$ region where valencelike IC would cause a large enhancement. Indeed, Fig. 5 shows that, after including the IC PDF shape predicted by BHPS with a mean momentum fraction of $1 \%$, the theory predictions are consistent with the data. Incorporating these novel forward $\mathcal{R}_{j}^{c}$ results into a global analysis should strongly constrain the large- $x$ charm PDF, both in size and in shape. While the large enhancement in the forwardmost $y(Z)$ interval is suggestive of valencelike IC, no definitive statements can be made until the $\mathcal{R}_{j}^{c}$ results are included in a global PDF analysis.

In conclusion, events containing a $Z$ boson and a charm jet are studied for the first time in the forward region of $p p$ collisions. The data sample used corresponds to an integrated luminosity of $6 \mathrm{fb}^{-1}$ collected at a center-ofmass energy of $13 \mathrm{TeV}$ with the LHCb detector. The ratio $\mathcal{R}_{j}^{c}$ is measured in intervals of $y(Z)$ and compared to NLO SM calculations. The observed spectrum exhibits a sizable enhancement at forward $Z$ rapidities, consistent with the effect expected if the proton wave function contains the $|u u d c \bar{c}\rangle$ component predicted by BHPS. However, conclusions about whether the proton contains valencelike intrinsic charm can only be drawn after incorporating these results into global PDF analyses.

We express our gratitude to our colleagues in the CERN accelerator departments for the excellent performance of the LHC. We thank the technical and administrative staff at the LHCb institutes. We acknowledge support from CERN and from the national agencies: CAPES, CNPq, FAPERJ, and FINEP (Brazil); MOST and NSFC (China); CNRS/ IN2P3 (France); BMBF, DFG, and MPG (Germany); INFN (Italy); NWO (Netherlands); MNiSW and NCN (Poland); MEN/IFA (Romania); MSHE (Russia); MICINN (Spain); SNSF and SER (Switzerland); NASU (Ukraine); STFC (United Kingdom); DOE NP and NSF (USA). We acknowledge the computing resources that are provided by CERN, IN2P3 (France), KIT and DESY (Germany), INFN (Italy), SURF (Netherlands), PIC (Spain), GridPP (United Kingdom), RRCKI and Yandex LLC (Russia), CSCS (Switzerland), IFIN-HH (Romania), CBPF (Brazil), PL-GRID (Poland), and NERSC (USA). We are indebted to the communities behind the multiple open-source software packages on which we depend. Individual groups or members have received support from ARC and ARDC (Australia); AvH Foundation (Germany); EPLANET, Marie Skłodowska-Curie Actions and ERC (European Union); $\mathrm{A}^{*} \mathrm{MIDEX}$, ANR, IPhU and Labex P2IO, and Région Auvergne-Rhône-Alpes (France); Key Research Program of Frontier Sciences of CAS, CAS PIFI, CAS CCEPP, Fundamental Research Funds for the Central Universities, and Sci. \& Tech. Program of Guangzhou (China); RFBR, RSF, and Yandex LLC (Russia); GVA, XuntaGal, and GENCAT (Spain); the Leverhulme Trust, the Royal Society, and UKRI (United Kingdom).

[1] S. J. Brodsky, A. Kusina, F. Lyonnet, I. Schienbein, H. Spiesberger, and R. Vogt, A review of the intrinsic heavy quark content of the nucleon, Adv. High Energy Phys. 2015, 231547 (2015).

[2] S. J. Brodsky, P. Hoyer, C. Peterson, and N. Sakai, The intrinsic charm of the proton, Phys. Lett. 93B, 451 (1980).

[3] S. J. Brodsky, C. Peterson, and N. Sakai, Intrinsic heavy quark states, Phys. Rev. D 23, 2745 (1981).

[4] E. Hoffmann and R. Moore, Subleading contributions to the intrinsic charm of the nucleon, Z. Phys. C 20, 71 (1983).

[5] F. S. Navarra, M. Nielsen, C. A. A. Nunes, and M. Teixeira, On the intrinsic charm component of the nucleon, Phys. Rev. D 54, 842 (1996).

[6] M. Franz, M. V. Polyakov, and K. Goeke, Heavy quark mass expansion and intrinsic charm in light hadrons, Phys. Rev. D 62, 074024 (2000).

[7] J. Pumplin, Light-cone models for intrinsic charm and bottom, Phys. Rev. D 73, 114015 (2006).

[8] W. Freeman and D. Toussaint (MILC Collaboration), Intrinsic strangeness and charm of the nucleon using improved staggered fermions, Phys. Rev. D 88, 054503 (2013). 
[9] S. Brodsky, G. de Teramond, and M. Karliner, Puzzles in hadronic physics and novel quantum chromodynamics phenomenology, Annu. Rev. Nucl. Part. Sci. 62, 1 (2012).

[10] M. Gong, A. Alexandru, Y. Chen, T. Doi, S. J. Dong, T. Draper, W. Freeman, M. Glatzmaier, A. Li, K. F. Liu, and Z. Liu (XQCD Collaboration), Strangeness and charmness content of the nucleon from overlap fermions on $2+1$ flavor domain-wall fermion configurations, Phys. Rev. D 88, 014503 (2013).

[11] T. J. Hobbs, J. T. Londergan, and W. Melnitchouk, Phenomenology of nonperturbative charm in the nucleon, Phys. Rev. D 89, 074008 (2014).

[12] R. D. Ball, V. Bertone, M. Bonvini, S. Forte, P. G. Merrild, J. Rojo, and L. Rottoli, Intrinsic charm in a matched generalmass scheme, Phys. Lett. B 754, 49 (2016).

[13] J. Blümlein, A kinematic condition on intrinsic charm, Phys. Lett. B 753, 619 (2016).

[14] S. Duan, C. S. An, and B. Saghai, Intrinsic charm content of the nucleon and charmness-nucleon sigma term, Phys. Rev. D 93, 114006 (2016).

[15] R. S. Sufian, T. Liu, A. Alexandru, S. J. Brodsky, G. F. de Téramond, H. G. Dosch, T. Draper, K.-F. Liu, and Y.-B. Yang, Constraints on charm-anticharm asymmetry in the nucleon from lattice QCD, Phys. Lett. B 808, 135633 (2020).

[16] M. G. Aartsen et al. (IceCube Collaboration), Evidence for high-energy extraterrestrial neutrinos at the IceCube detector, Science 342, 1242856 (2013).

[17] R. Gauld, J. Rojo, L. Rottoli, S. Sarkar, and J. Talbert, The prompt atmospheric neutrino flux in the light of $\mathrm{LHCb}$, J. High Energy Phys. 02 (2016) 130.

[18] F. Halzen and L. Wille, Charm contribution to the atmospheric neutrino flux, Phys. Rev. D 94, 014014 (2016).

[19] R. Laha and S. J. Brodsky, IceCube can constrain the intrinsic charm of the proton, Phys. Rev. D 96, 123002 (2017).

[20] A. V. Giannini, V. P. Gonçalves, and F. S. Navarra, Intrinsic charm contribution to the prompt atmospheric neutrino flux, Phys. Rev. D 98, 014012 (2018).

[21] V. P. Gonalves, R. Maciula, and A. Szczurek, Impact of intrinsic charm amount in the nucleon and saturation effects on the prompt atmospheric $\nu_{\mu}$ flux for IceCube, arXiv: 2103.05503.

[22] H. L. Lai, P. Nadolsky, J. Pumplin, D. Stump, W.-K. Tung, and C.-P. Yuan, The Strange parton distribution of the nucleon: Global analysis and applications, J. High Energy Phys. 04 (2007) 089.

[23] S. J. Brodsky, A. S. Goldhaber, B. Z. Kopeliovich, and I. Schmidt, Higgs hadroproduction at large Feynman $x$, Nucl. Phys. B807, 334 (2009).

[24] T. P. Stavreva and J. F. Owens, Direct photon production in association with a heavy quark at hadron colliders, Phys. Rev. D 79, 054017 (2009).

[25] V. A. Bednyakov, M. A. Demichev, G. I. Lykasov, T. Stavreva, and M. Stockton, Searching for intrinsic charm in the proton at the LHC, Phys. Lett. B 728, 602 (2014).

[26] S. Dulat, T. J. Hou, J. Gao, J. Huston, J. Pumplin, C. Schmidt, D. Stump, and C. P. Yuan, Intrinsic charm parton distribution functions from CTEQ-TEA global analysis, Phys. Rev. D 89, 073004 (2014).
[27] F. Halzen, Y. S. Jeong, and C. S. Kim, Charge asymmetry of weak boson production at the LHC and the charm content of the proton, Phys. Rev. D 88, 073013 (2013).

[28] S. Rostami, A. Khorramian, A. Aleedaneshvar, and M. Goharipour, The impact of the intrinsic charm quark content of a proton on the differential $\gamma+c$ cross section, J. Phys. G 43, 055001 (2016).

[29] T. Boettcher, P. Ilten, and M. Williams, Direct probe of the intrinsic charm content of the proton, Phys. Rev. D 93, 074008 (2016).

[30] G. Bailas and V. P. Goncalves, Phenomenological implications of the intrinsic charm in the $Z$ boson production at the LHC, Eur. Phys. J. C 76, 105 (2016).

[31] A. V. Lipatov, G. I. Lykasov, Y. Y. Stepanenko, and V. A. Bednyakov, Probing proton intrinsic charm in photon or $Z$ boson production accompanied by heavy jets at the LHC, Phys. Rev. D 94, 053011 (2016).

[32] W. Bai and M. H. Reno, Prompt neutrinos and intrinsic charm at SHiP, J. High Energy Phys. 02 (2019) 077.

[33] J. J. Aubert et al. (European Muon Collaboration), Production of charmed particles in $250-\mathrm{GeV} \mu^{+}$-iron interactions, Nucl. Phys. B213, 31 (1983).

[34] R. Aaij et al. (LHCb Collaboration), First Measurement of Charm Production in Fixed-Target Configuration at the LHC, Phys. Rev. Lett. 122, 132002 (2019).

[35] B. W. Harris, J. Smith, and R. Vogt, Reanalysis of the EMC charm production data with extrinsic and intrinsic charm at NLO, Nucl. Phys. B461, 181 (1996).

[36] F. M. Steffens, W. Melnitchouk, and A. W. Thomas, Charm in the nucleon, Eur. Phys. J. C 11, 673 (1999).

[37] P. Jimenez-Delgado, T. J. Hobbs, J. T. Londergan, and W. Melnitchouk, New Limits on Intrinsic Charm in the Nucleon from Global Analysis of Parton Distributions, Phys. Rev. Lett. 114, 082002 (2015).

[38] T.-J. Hou, S. Dulat, J. Gao, M. Guzzi, J. Huston, P. Nadolsky, C. Schmidt, J. Winter, K. Xie, and C.-P. Yuan, CT14 intrinsic charm parton distribution functions from CTEQ-TEA global analysis, J. High Energy Phys. 02 (2018) 059.

[39] R. D. Ball, V. Bertone, M. Bonvini, S. Carrazza, S. Forte, A. Guffanti, N. P. Hartland, J. Rojo, and L. Rottoli (NNPDF Collaboration), A determination of the charm content of the proton, Eur. Phys. J. C 76, 647 (2016).

[40] V. M. Abazov et al. (D0 Collaboration), Measurement of Associated Production of Z Bosons with Charm Quark Jets in $p \bar{p}$ Collisions at $\sqrt{s}=1.96 \mathrm{TeV}$, Phys. Rev. Lett. 112, 042001 (2014).

[41] A. M. Sirunyan et al. (CMS Collaboration), Measurement of the associated production of a $Z$ boson with charm or bottom quark jets in proton-proton collisions at $\sqrt{\mathrm{s}}=$ 13 TeV, Phys. Rev. D 102, 032007 (2020).

[42] J. Butterworth et al., PDF4LHC recommendations for LHC Run II, J. Phys. G 43, 023001 (2016).

[43] See Supplemental Material at http://link.aps.org/ supplemental/10.1103/PhysRevLett.128.082001 for details on the theoretical predictions, and for additional plots and numerical results.

[44] M. Cacciari, G. P. Salam, and G. Soyez, The anti- $k_{T}$ jet clustering algorithm, J. High Energy Phys. 04 (2008) 063.

[45] R. Aaij et al. (LHCb Collaboration), Identification of charm jets at LHCb, arXiv:2112.08435. 
[46] A. A. Alves Jr. et al. (LHCb Collaboration), The LHCb detector at the LHC, J. Instrum. 3, S08005 (2008).

[47] R. Aaij et al. (LHCb Collaboration), LHCb detector performance, Int. J. Mod. Phys. A 30, 1530022 (2015).

[48] T. Sjöstrand, S. Mrenna, and P. Skands, A brief introduction to PYTHIA8.1, Comput. Phys. Commun. 178, 852 (2008); T. Sjöstrand, S. Mrenna, and P. Skands, PYTHIA6.4 physics and manual, J. High Energy Phys. 05 (2006) 026.

[49] I. Belyaev et al., Handling of the generation of primary events in Gauss, the LHCb simulation framework, J. Phys. Conf. Ser. 331, 032047 (2011).

[50] D. J. Lange, The EvtGen particle decay simulation package, Nucl. Instrum. Methods Phys. Res., Sect. A 462, 152 (2001).

[51] N. Davidson, T. Przedzinski, and Z. Was, PHOTOS interface in $\mathrm{C}++$ : Technical and physics documentation, Comput. Phys. Commun. 199, 86 (2016).

[52] J. Allison et al. (Geant4 Collaboration), GEANT4 developments and applications, IEEE Trans. Nucl. Sci. 53, 270 (2006); S. Agostinelli et al. (Geant4 Collaboration), GEANT4: A simulation toolkit, Nucl. Instrum. Methods Phys. Res., Sect. A 506, 250 (2003).

[53] M. Clemencic, G. Corti, S. Easo, C. R. Jones, S. Miglioranzi, M. Pappagallo, and P. Robbe, The LHCb simulation application, Gauss: Design, evolution and experience, J. Phys. Conf. Ser. 331, 032023 (2011).

[54] R. Aaij et al., The LHCb trigger and its performance in 2011, J. Instrum. 8, P04022 (2013).

[55] R. Aaij et al., Performance of the LHCb trigger and full realtime reconstruction in Run 2 of the LHC, J. Instrum. 14, P04013 (2019).

[56] R. Aaij et al. (LHCb Collaboration), Study of forward $Z+$ jet production in $p p$ collisions at $\sqrt{s}=7 \mathrm{TeV}$, J. High Energy Phys. 01 (2014) 033.

[57] M. Cacciari, G. P. Salam, and G. Soyez, FastJet user manual, Eur. Phys. J. C 72, 1896 (2012).

[58] R. Aaij et al. (LHCb Collaboration), Identification of beauty and charm quark jets at LHCb, J. Instrum. 10, P06013 (2015).

[59] R. Aaij et al. (LHCb Collaboration), First Observation of Top Quark Production in the Forward Region, Phys. Rev. Lett. 115, 112001 (2015).

[60] R. Aaij et al. (LHCb Collaboration), Study of $W$ boson production in association with beauty and charm, Phys. Rev. D 92, 052012 (2015).

[61] G. D'Agostini, A multidimensional unfolding method based on Bayes' theorem, Nucl. Instrum. Methods Phys. Res., Sect. A 362, 487 (1995).

[62] T. Adye, Unfolding algorithms and tests using RooUnfold, in Proceedings of the PHYSTAT 2011 (CERN, Geneva, 2011), pp. 313-318.

[63] M. Alexander et al., Mapping the material in the $\mathrm{LHCb}$ vertex locator using secondary hadronic interactions, J. Instrum. 13, P06008 (2018).

[64] R. Aaij et al. (LHCb Collaboration), Study of $J / \psi$ Production in Jets, Phys. Rev. Lett. 118, 192001 (2017).

[65] P. A. Zyla et al. (Particle Data Group), Review of particle physics, Prog. Theor. Exp. Phys. 2020, 083C01 (2020).
[66] M. Lisovyi, A. Verbytskyi, and O. Zenaiev, Combined analysis of charm-quark fragmentation-fraction measurements, Eur. Phys. J. C 76, 397 (2016).

[67] S. Alioli, P. Nason, C. Oleari, and E. Re, Vector boson plus one jet production in POWHEG, J. High Energy Phys. 01 (2011) 095.

[68] T. Sjöstrand, S. Ask, J. R. Christiansen, R. Corke, N. Desai, P. Ilten, S. Mrenna, S. Prestel, C. O. Rasmussen, and P.Z. Skands, An introduction to PYTHIA8.2, Comput. Phys. Commun. 191, 159 (2015).

[69] P. Nason, A new method for combining NLO QCD with shower Monte Carlo algorithms, J. High Energy Phys. 11 (2004) 040.

[70] J. Alwall, R. Frederix, S. Frixione, V. Hirschi, F. Maltoni, O. Mattelaer, H.-S. Shao, T. Stelzer, P. Torrielli, and M. Zaro, The automated computation of tree-level and next-toleading order differential cross sections, and their matching to parton shower simulations, J. High Energy Phys. 07 (2014) 079.

[71] R. Frederix and S. Frixione, Merging meets matching in MC@NLO, J. High Energy Phys. 12 (2012) 061.

[72] P. Golonka and Z. Was, PHOTOS Monte Carlo: A precision tool for QED corrections in $Z$ and $W$ decays, Eur. Phys. J. C 45, 97 (2006).

[73] W. T. Giele and S. Keller, Implications of hadron collider observables on parton distribution function uncertainties, Phys. Rev. D 58, 094023 (1998).

[74] J. M. Campbell, R. K. Ellis, and C. Williams, Vector boson pair production at the LHC, J. High Energy Phys. 07 (2011) 018.

[75] J. M. Campbell, R. K. Ellis, and W. T. Giele, A multithreaded version of MCFM, Eur. Phys. J. C 75, 246 (2015).

[76] R. D. Ball, V. Bertone, S. Carrazza, C. S. Deans, L. D. Debbio, S. Forte, A. Guffanti, N. P. Hartland, J. I. Latorre, J. Rojo, and M. Ubiali (NNPDF Collaboration), Parton distributions for the LHC Run II, J. High Energy Phys. 04 (2015) 040.

[77] L. A. Harland-Lang, A. D. Martin, P. Motylinski, and R. S. Thorne, Parton distributions in the LHC era: MMHT 2014 PDFs, Eur. Phys. J. C 75, 204 (2015).

[78] R. Gauld, A. G. Ridder, E. W. N. Glover, A. Huss, and I. Majer, Predictions for $Z$-Boson Production in Association with a $b$-Jet at $\mathcal{O}\left(\alpha_{s}^{3}\right)$, Phys. Rev. Lett. 125, 222002 (2020).

[79] S. Dulat, T.-J. Hou, J. Gao, M. Guzzi, J. Huston, P. Nadolsky, J. Pumplin, C. Schmidt, D. Stump, and C.-P. Yuan, New parton distribution functions from a global analysis of quantum chromodynamics, Phys. Rev. D 93, 033006 (2016).

[80] S. Alekhin, J. Blümlein, and S. Moch, NLO PDFs from the ABMP16 fit, Eur. Phys. J. C 78, 477 (2018).

[81] P. Jimenez-Delgado and E. Reya, Delineating parton distributions and the strong coupling, Phys. Rev. D 89, 074049 (2014).

[82] H. Abramowicz et al. (H1, ZEUS Collaborations), Combination of measurements of inclusive deep inelastic $e^{ \pm} p$ scattering cross sections and QCD analysis of HERA data, Eur. Phys. J. C 75, 580 (2015). 
R. Aaij, ${ }^{32}$ A. S. W. Abdelmotteleb, ${ }^{56}$ C. Abellán Beteta, ${ }^{50}$ F. J. Abudinen Gallego, ${ }^{56}$ T. Ackernley, ${ }^{60}$ B. Adeva, ${ }^{46}$ M. Adinolfi, ${ }^{54}$ H. Afsharnia, ${ }^{9}$ C. Agapopoulou, ${ }^{13}$ C. A. Aidala, ${ }^{87}$ S. Aiola, ${ }^{25}$ Z. Ajaltouni, ${ }^{9}$ S. Akar, ${ }^{65}$ J. Albrecht, ${ }^{15}$ F. Alessio, ${ }^{48}$ M. Alexander, ${ }^{59}$ A. Alfonso Albero, ${ }^{45}$ Z. Aliouche, ${ }^{62}$ G. Alkhazov, ${ }^{38}$ P. Alvarez Cartelle, ${ }^{55}$ S. Amato, ${ }^{2}$ J. L. Amey, ${ }^{54}$ Y. Amhis, ${ }^{11}$ L. An,${ }^{48}$ L. Anderlini, ${ }^{22}$ A. Andreianov, ${ }^{38}$ M. Andreotti, ${ }^{21}$ F. Archilli, ${ }^{17}$ A. Artamonov, ${ }^{44}$ M. Artuso, ${ }^{68}$ K. Arzymatov, ${ }^{42}$ E. Aslanides, ${ }^{10}$ M. Atzeni, ${ }^{50}$ B. Audurier, ${ }^{12}$ S. Bachmann, ${ }^{17}$ M. Bachmayer, ${ }^{49}$ J. J. Back, ${ }^{56}$ P. Baladron Rodriguez, ${ }^{46}$ V. Balagura, ${ }^{12}$ W. Baldini, ${ }^{21}$ J. Baptista Leite, ${ }^{1}$ M. Barbetti, ${ }^{22}$ R. J. Barlow, ${ }^{62}$ S. Barsuk, ${ }^{11}$ W. Barter ${ }^{61}$ M. Bartolini, ${ }^{24, b}$ F. Baryshnikov, ${ }^{83}$ J. M. Basels, ${ }^{14}$ S. Bashir,${ }^{34}$ G. Bassi, ${ }^{29}$ B. Batsukh, ${ }^{68}$ A. Battig, ${ }^{15}$ A. Bay, ${ }^{49}$ A. Beck, ${ }^{56}$ M. Becker, ${ }^{15}$ F. Bedeschi, ${ }^{29}$ I. Bediaga, ${ }^{1}$ A. Beiter ${ }^{68}$ V. Belavin, ${ }^{42}$ S. Belin, ${ }^{27}$ V. Bellee,${ }^{50}$ K. Belous, ${ }^{44}$ I. Belov, ${ }^{40}$ I. Belyaev, ${ }^{41}$ G. Bencivenni, ${ }^{23}$ E. Ben-Haim, ${ }^{13}$ A. Berezhnoy, ${ }^{40}$ R. Bernet, ${ }^{50}$ D. Berninghoff, ${ }^{17}$ H. C. Bernstein, ${ }^{68}$ C. Bertella, ${ }^{48}$ A. Bertolin, ${ }^{28}$ C. Betancourt, ${ }^{50}$ F. Betti, ${ }^{48}$ Ia. Bezshyiko, ${ }^{50}$ S. Bhasin, ${ }^{54}$ J. Bhom, ${ }^{35}$ L. Bian, ${ }^{73}$ M. S. Bieker,${ }^{15}$ S. Bifani ${ }^{53}$ P. Billoir, ${ }^{13}$ M. Birch, ${ }^{61}$ F. C. R. Bishop, ${ }^{55}$ A. Bitadze,${ }^{62}$ A. Bizzeti, ${ }^{22, c}$ M. Bjørn, ${ }^{63}$ M. P. Blago, ${ }^{48}$ T. Blake,${ }^{56}$ F. Blanc, ${ }^{49}$ S. Blusk, ${ }^{68}$ D. Bobulska, ${ }^{59}$ J. A. Boelhauve, ${ }^{15}$ O. Boente Garcia, ${ }^{46}$ T. Boettcher, ${ }^{65}$ A. Boldyrev, ${ }^{82}$ A. Bondar ${ }^{43}$ N. Bondar, ${ }^{38,48}$ S. Borghi, ${ }^{62}$ M. Borisyak, ${ }^{42}$ M. Borsato, ${ }^{17}$ J. T. Borsuk, ${ }^{35}$ S. A. Bouchiba, ${ }^{49}$ T. J. V. Bowcock, ${ }^{60}$ A. Boyer, ${ }^{48}$ C. Bozzi, ${ }^{21}$ M. J. Bradley, ${ }^{61}$ S. Braun ${ }^{66}$ A. Brea Rodriguez,${ }^{46}$ J. Brodzicka, ${ }^{35}$ A. Brossa Gonzalo, ${ }^{56}$ D. Brundu, ${ }^{27}$ A. Buonaura, ${ }^{50}$ L. Buonincontri, ${ }^{28}$ A. T. Burke, ${ }^{62}$ C. Burr, ${ }^{48}$ A. Bursche ${ }^{72}$ A. Butkevich, ${ }^{39}$ J. S. Butter, ${ }^{32}$ J. Buytaert, ${ }^{48}$ W. Byczynski, ${ }^{48}$ S. Cadeddu, ${ }^{27}$ H. Cai, ${ }^{73}$ R. Calabrese, ${ }^{21, d}$ L. Calefice, ${ }^{15}{ }^{13}$ L. Calero Diaz, ${ }^{23}$ S. Cali, ${ }^{23}$ R. Calladine, ${ }^{53}$ M. Calvi, ${ }^{26, e}$ M. Calvo Gomez, ${ }^{85}$ P. Camargo Magalhaes,${ }^{54}$ P. Campana, ${ }^{23}$ A. F. Campoverde Quezada, ${ }^{6}$ S. Capelli, ${ }^{26, e}$ L. Capriotti ${ }^{20, f}$ A. Carbone, ${ }^{20, f}$ G. Carboni, ${ }^{31}$ R. Cardinale, ${ }^{24, b}$ A. Cardini, ${ }^{27}$ I. Carli, ${ }^{4}$ P. Carniti, ${ }^{26, e}$ L. Carus, ${ }^{14}$ K. Carvalho Akiba, ${ }^{32}$ A. Casais Vidal, ${ }^{46}$ G. Casse, ${ }^{60}$ M. Cattaneo,${ }^{48}$ G. Cavallero, ${ }^{48}$ S. Celani, ${ }^{49}$ J. Cerasoli, ${ }^{10}$ D. Cervenkov, ${ }^{63}$ A. J. Chadwick, ${ }^{60}$ M. G. Chapman, ${ }^{54}$ M. Charles, ${ }^{13} \mathrm{Ph}$. Charpentier, ${ }^{48}$ G. Chatzikonstantinidis, ${ }^{53}$ C. A. Chavez Barajas, ${ }^{60}$ M. Chefdeville, ${ }^{8}$ C. Chen, ${ }^{3}$ S. Chen, ${ }^{4}$ A. Chernov, ${ }^{35}$ V. Chobanova, ${ }^{46}$ S. Cholak, ${ }^{49}$ M. Chrzaszcz, ${ }^{35}$ A. Chubykin ${ }^{38}$ V. Chulikov, ${ }^{38}$ P. Ciambrone, ${ }^{23}$ M. F. Cicala, ${ }^{56}$ X. Cid Vidal, ${ }^{46}$ G. Ciezarek, ${ }^{48}$ P. E. L. Clarke, ${ }^{58}$ M. Clemencic, ${ }^{48}$ H. V. Cliff, ${ }^{55}$ J. Closier, ${ }^{48}$ J. L. Cobbledick, ${ }^{62}$ V. Coco, ${ }^{48}$ J. A. B. Coelho, ${ }^{11}$ J. Cogan, ${ }^{10}$ E. Cogneras, ${ }^{9}$ L. Cojocariu, ${ }^{37}$ P. Collins, ${ }^{48}$ T. Colombo, ${ }^{48}$ L. Congedo, ${ }^{19,9}$ A. Contu, ${ }^{27}$ N. Cooke, ${ }^{53}$ G. Coombs, ${ }^{59}$ I. Corredoira, ${ }^{46}$ G. Corti, ${ }^{48}$ C. M. Costa Sobral,${ }^{56}$ B. Couturier, ${ }^{48}$ D. C. Craik,${ }^{64}$ J. Crkovská, ${ }^{67}$ M. Cruz Torres, ${ }^{1}$ R. Currie, ${ }^{58}$ C. L. Da Silva, ${ }^{67}$ S. Dadabaev, ${ }^{83}$ L. Dai, ${ }^{71}$ E. Dall'Occo, ${ }^{15}$ J. Dalseno, ${ }^{46}$ C. D'Ambrosio, ${ }^{48}$ A. Danilina, ${ }^{41}$ P. d'Argent, ${ }^{48}$ J. E. Davies, ${ }^{62}$ A. Davis, ${ }^{62}$ O. De Aguiar Francisco, ${ }^{62}$ K. De Bruyn, ${ }^{79}$ S. De Capua ${ }^{62}$ M. De Cian, ${ }^{49}$ J. M. De Miranda, ${ }^{1}$ L. De Paula, ${ }^{2}$ M. De Serio, ${ }^{19, \mathrm{~g}}$ D. De Simone, ${ }^{50}$ P. De Simone, ${ }^{23}$ F. De Vellis, ${ }^{15}$ J. A. de Vries,${ }^{80}$ C. T. Dean, ${ }^{67}$ F. Debernardis, ${ }^{19, g}$ D. Decamp, ${ }^{8}$ V. Dedu, ${ }^{10}$ L. Del Buono, ${ }^{13}$ B. Delaney, ${ }^{55}$ H.-P. Dembinski, ${ }^{15}$ A. Dendek,${ }^{34}$ V. Denysenko, ${ }^{50}$ D. Derkach, ${ }^{82}$ O. Deschamps, ${ }^{9}$ F. Desse, ${ }^{11}$ F. Dettori, ${ }^{27, h}$ B. Dey, ${ }^{77}$ A. Di Cicco, ${ }^{23}$ P. Di Nezza, ${ }^{23}$ S. Didenko, ${ }^{83}$ L. Dieste Maronas, ${ }^{46}$ H. Dijkstra, ${ }^{48}$ V. Dobishuk, ${ }^{52}$ C. Dong, ${ }^{3}$ A. M. Donohoe, ${ }^{18}$ F. Dordei, ${ }^{27}$ A. C. dos Reis, ${ }^{1}$ L. Douglas, ${ }^{59}$ A. Dovbnya,${ }^{51}$ A. G. Downes, ${ }^{8}$ M. W. Dudek, ${ }^{35}$ L. Dufour,${ }^{48}$ V. Duk,${ }^{78}$ P. Durante, ${ }^{48}$ J. M. Durham, ${ }^{67}$ D. Dutta, ${ }^{62}$ A. Dziurda, ${ }^{35}$ A. Dzyuba, ${ }^{38}$ S. Easo, ${ }^{57}$ U. Egede ${ }^{69}$ V. Egorychev, ${ }^{41}$ S. Eidelman, ${ }^{43, a, i}$ S. Eisenhardt,${ }^{58}$ S. Ek-In, ${ }^{49}$ L. Eklund, ${ }^{59,86}$ S. Ely, ${ }^{68}$ A. Ene, ${ }^{37}$ E. Epple, ${ }^{67}$ S. Escher ${ }^{14}$ J. Eschle, ${ }^{50}$ S. Esen, ${ }^{13}$ T. Evans, ${ }^{48}$ A. Falabella, ${ }^{20}$ J. Fan, ${ }^{3}$ Y. Fan, ${ }^{6}$ B. Fang, ${ }^{73}$ S. Farry, ${ }^{60}$ D. Fazzini, ${ }^{26, \mathrm{e}}$ M. Féo, ${ }^{48}$ A. Fernandez Prieto, ${ }^{46}$ A. D. Fernez, ${ }^{66}$ F. Ferrari, ${ }^{20, f}$ L. Ferreira Lopes,${ }^{49}$ F. Ferreira Rodrigues, ${ }^{2}$ S. Ferreres Sole ${ }^{32}$ M. Ferrillo, ${ }^{50}$ M. Ferro-Luzzi, ${ }^{48}$ S. Filippov, ${ }^{39}$ R. A. Fini, ${ }^{19}$ M. Fiorini, ${ }^{21, d}$ M. Firlej, ${ }^{34}$ K. M. Fischer ${ }^{63}$ D. S. Fitzgerald ${ }^{87}$ C. Fitzpatrick, ${ }^{62}$ T. Fiutowski, ${ }^{34}$ A. Fkiaras, ${ }^{48}$ F. Fleuret,,${ }^{12}$ M. Fontana, ${ }^{13}$ F. Fontanelli, ${ }^{24, b}$ R. Forty, ${ }^{48}$ D. Foulds-Holt,${ }^{55}$ V. Franco Lima, ${ }^{60}$ M. Franco Sevilla, ${ }^{66}$ M. Frank,${ }^{48}$ E. Franzoso,${ }^{21}$ G. Frau, ${ }^{17}$ C. Frei, ${ }^{48}$ D. A. Friday, ${ }^{59}$ J. Fu, ${ }^{6}$ Q. Fuehring, ${ }^{15}$ E. Gabriel, ${ }^{32}$ G. Galati, ${ }^{19, g}$ A. Gallas Torreira, ${ }^{46}$ D. Galli, ${ }^{20, f}$ S. Gambetta, ${ }^{58,48}$ Y. Gan, ${ }^{3}$ M. Gandelman, ${ }^{2}$ P. Gandini, ${ }^{25}$ Y. Gao, ${ }^{5}$ M. Garau, ${ }^{27}$ L. M. Garcia Martin, ${ }^{56}$ P. Garcia Moreno, ${ }^{45}$ J. García Pardiñas, ${ }^{26, e}$ B. Garcia Plana, ${ }^{46}$ F. A. Garcia Rosales, ${ }^{12}$ L. Garrido, ${ }^{45}$ C. Gaspar, ${ }^{48}$ R. E. Geertsema, ${ }^{32}$ D. Gerick, ${ }^{17}$ L. L. Gerken, ${ }^{15}$ E. Gersabeck, ${ }^{62}$ M. Gersabeck, ${ }^{62}$ T. Gershon, ${ }^{56}$ D. Gerstel, ${ }^{10}$ L. Giambastiani, ${ }^{28}$ V. Gibson, ${ }^{55}$ H. K. Giemza, ${ }^{36}$

A. L. Gilman, ${ }^{63}$ M. Giovannetti, ${ }^{23, j}$ A. Gioventù, ${ }^{46}$ P. Gironella Gironell, ${ }^{45}$ L. Giubega,${ }^{37}$ C. Giugliano, ${ }^{21,48, d}$ K. Gizdov, ${ }^{58}$ E. L. Gkougkousis, ${ }^{48}$ V. V. Gligorov,${ }^{13}$ C. Göbel, ${ }^{70}$ E. Golobardes,${ }^{85}$ D. Golubkov, ${ }^{41}$ A. Golutvin, ${ }^{61,83}$ A. Gomes, ${ }^{1, k}$ S. Gomez Fernandez, ${ }^{45}$ F. Goncalves Abrantes, ${ }^{63}$ M. Goncerz, ${ }^{35}$ G. Gong, ${ }^{3}$ P. Gorbounov, ${ }^{41}$ I. V. Gorelov,${ }^{40}$ C. Gotti, ${ }^{26}$ E. Govorkova, ${ }^{48}$ J. P. Grabowski, ${ }^{17}$ T. Grammatico, ${ }^{13}$ L. A. Granado Cardoso, ${ }^{48}$ E. Graugés,${ }^{45}$ E. Graverini, ${ }^{49}$ G. Graziani, ${ }^{22}$ A. Grecu, ${ }^{37}$ L. M. Greeven, ${ }^{32}$ N. A. Grieser, ${ }^{4}$ L. Grillo, ${ }^{62}$ S. Gromov,${ }^{83}$ B. R. Gruberg Cazon,${ }^{63}$ C. Gu, ${ }^{3}$ M. Guarise, ${ }^{21}$ M. Guittiere, ${ }^{11}$ P. A. Günther, ${ }^{17}$ E. Gushchin, ${ }^{39}$ A. Guth, ${ }^{14}$ Y. Guz,${ }^{44}$ T. Gys,${ }^{48}$ T. Hadavizadeh, ${ }^{69}$ G. Haefeli, ${ }^{49}$ C. Haen, ${ }^{48}$ J. Haimberger, ${ }^{48}$ T. Halewood-leagas, ${ }^{60}$ P. M. Hamilton, ${ }^{66}$ J. P. Hammerich, ${ }^{60}$ Q. Han, ${ }^{7}$ X. Han, ${ }^{17}$ T. H. Hancock, ${ }^{63}$ 
E. B. Hansen, ${ }^{62}$ S. Hansmann-Menzemer, ${ }^{17}$ N. Harnew, ${ }^{63}$ T. Harrison, ${ }^{60}$ C. Hasse, ${ }^{48}$ M. Hatch, ${ }^{48}$ J. He, ${ }^{6,1}$ M. Hecker, ${ }^{61}$ K. Heijhoff, ${ }^{32}$ K. Heinicke, ${ }^{15}$ A. M. Hennequin, ${ }^{48}$ K. Hennessy, ${ }^{60}$ L. Henry, ${ }^{48}$ J. Heuel, ${ }^{14}$ A. Hicheur, ${ }^{2}$ D. Hill, ${ }^{49}$ M. Hilton, ${ }^{62}$ S. E. Hollitt, ${ }^{15}$ R. Hou,${ }^{7}$ Y. Hou, ${ }^{8}$ J. Hu, ${ }^{17}$ J. Hu, ${ }^{72}$ W. Hu,,${ }^{7}$ X. Hu, ${ }^{3}$ W. Huang,,${ }^{6}$ X. Huang, ${ }^{73}$ W. Hulsbergen, ${ }^{32}$ R. J. Hunter, ${ }^{56}$ M. Hushchyn, ${ }^{82}$ D. Hutchcroft,,${ }^{60}$ D. Hynds, ${ }^{32}$ P. Ibis, ${ }^{15}$ M. Idzik, ${ }^{34}$ D. Ilin, ${ }^{38}$ P. Ilten, ${ }^{65}$ A. Inglessi, ${ }^{38}$ A. Ishteev, ${ }^{83}$ K. Ivshin, ${ }^{38}$ R. Jacobsson, ${ }^{48}$ H. Jage, ${ }^{14}$ S. Jakobsen, ${ }^{48}$ E. Jans, ${ }^{32}$ B. K. Jashal, ${ }^{47}$ A. Jawahery, ${ }^{66}$ V. Jevtic, ${ }^{15}$ F. Jiang, ${ }^{3}$ M. John, ${ }^{63}$ D. Johnson, ${ }^{48}$ C. R. Jones,${ }^{55}$ T. P. Jones, ${ }^{56}$ B. Jost ${ }^{48}$ N. Jurik, ${ }^{48}$ S. H. Kalavan Kadavath, ${ }^{34}$ S. Kandybei, ${ }^{51}$ Y. Kang, ${ }^{3}$ M. Karacson, ${ }^{48}$ M. Karpov, ${ }^{82}$ F. Keizer, ${ }^{48}$ D. M. Keller, ${ }^{68}$ M. Kenzie,${ }^{56}$ T. Ketel,${ }^{33}$ B. Khanji, ${ }^{15}$ A. Kharisova, ${ }^{84}$ S. Kholodenko, ${ }^{44}$ T. Kirn, ${ }^{14}$ V. S. Kirsebom, ${ }^{49}$ O. Kitouni, ${ }^{64}$ S. Klaver, ${ }^{32}$ N. Kleijne, ${ }^{29}$ K. Klimaszewski, ${ }^{36}$ M. R. Kmiec, ${ }^{36}$ S. Koliiev, ${ }^{52}$ A. Kondybayeva, ${ }^{83}$ A. Konoplyannikov, ${ }^{41}$ P. Kopciewicz, ${ }^{34}$ R. Kopecna, ${ }^{17}$ P. Koppenburg, ${ }^{32}$ M. Korolev, ${ }^{40}$ I. Kostiuk, ${ }^{32,52}$ O. Kot, ${ }^{52}$ S. Kotriakhova, ${ }^{21,38}$ P. Kravchenko, ${ }^{38}$ L. Kravchuk, ${ }^{39}$ R. D. Krawczyk, ${ }^{48}$ M. Kreps, ${ }^{56}$ F. Kress ${ }^{61}$ S. Kretzschmar, ${ }^{14}$ P. Krokovny, ${ }^{43, i}$ W. Krupa,${ }^{34}$ W. Krzemien, ${ }^{36}$ M. Kucharczyk,${ }^{35}$ V. Kudryavtsev, ${ }^{43, i}$ H. S. Kuindersma, ${ }^{32,33}$ G. J. Kunde, ${ }^{67}$ T. Kvaratskheliya, ${ }^{41}$ D. Lacarrere, ${ }^{48}$ G. Lafferty, ${ }^{62}$ A. Lai, ${ }^{27}$ A. Lampis, ${ }^{27}$ D. Lancierini, ${ }^{50}$ J. J. Lane, ${ }^{62}$ R. Lane ${ }^{54}$ G. Lanfranchi, ${ }^{23}$ C. Langenbruch, ${ }^{14}$ J. Langer,${ }^{15}$ O. Lantwin, ${ }^{83}$ T. Latham, ${ }^{56}$ F. Lazzari, ${ }^{29, m}$ R. Le Gac, ${ }^{10}$ S. H. Lee, ${ }^{87}$ R. Lefèvre, ${ }^{9}$ A. Leflat, ${ }^{40}$ S. Legotin, ${ }^{83}$ O. Leroy, ${ }^{10}$ T. Lesiak, ${ }^{35}$ B. Leverington, ${ }^{17} \mathrm{H} . \mathrm{Li},{ }^{72} \mathrm{P} . \mathrm{Li},{ }^{17} \mathrm{~S} . \mathrm{Li}^{7}{ }^{7} \mathrm{Y} . \mathrm{Li}^{4}{ }^{4}$ Y. Li ${ }^{4}$ Z. Li ${ }^{68}$ X. Liang, ${ }^{68}$ T. Lin,${ }^{61}$ R. Lindner, ${ }^{48}$ V. Lisovskyi, ${ }^{15}$ R. Litvinov, ${ }^{27}$ G. Liu, ${ }^{72}$ H. Liu, ${ }^{6}$ Q. Liu, ${ }^{6}$ S. Liu, ${ }^{4}$ A. Lobo Salvia, ${ }^{45}$ A. Loi, ${ }^{27}$ J. Lomba Castro, ${ }^{46}$ I. Longstaff, ${ }^{59}$ J. H. Lopes, ${ }^{2}$ S. Lopez Solino, ${ }^{46}$ G. H. Lovell, ${ }^{55}$ Y. Lu, ${ }^{4}$ C. Lucarelli, ${ }^{22}$ D. Lucchesi, ${ }^{28, \mathrm{n}}$ S. Luchuk ${ }^{39}$ M. Lucio Martinez, ${ }^{32}$ V. Lukashenko, ${ }^{32,52}$ Y. Luo, ${ }^{3}$ A. Lupato, ${ }^{62}$ E. Luppi, ${ }^{21, \mathrm{~d}}$ O. Lupton, ${ }^{56}$ A. Lusiani, ${ }^{29,0}$ X. Lyu,${ }^{6}$ L. Ma, ${ }^{4}$ R. Ma, ${ }^{6}$ S. Maccolini, ${ }^{20, f}$ F. Machefert, ${ }^{11}$ F. Maciuc, ${ }^{37}$ V. Macko, ${ }^{49}$ P. Mackowiak, ${ }^{15}$ S. Maddrell-Mander,${ }^{54}$ O. Madejczyk ${ }^{34}$ L. R. Madhan Mohan,${ }^{54}$ O. Maev, ${ }^{38}$ A. Maevskiy, ${ }^{82}$ D. Maisuzenko, ${ }^{38}$ M. W. Majewski, ${ }^{34}$ J. J. Malczewski, ${ }^{35}$ S. Malde, ${ }^{63}$ B. Malecki, ${ }^{48}$ A. Malinin,${ }^{81}$ T. Maltsev, ${ }^{43, i}$ H. Malygina, ${ }^{17}$ G. Manca, ${ }^{27, h}$ G. Mancinelli, ${ }^{10}$ D. Manuzzi, ${ }^{20, \mathrm{f}}$ D. Marangotto, ${ }^{25, p}$ J. Maratas, ${ }^{9, \mathrm{q}}$ J. F. Marchand, ${ }^{8}$ U. Marconi, ${ }^{20}$ S. Mariani, ${ }^{22, r}$ C. Marin Benito, ${ }^{48}$ M. Marinangeli, ${ }^{49}$ J. Marks, ${ }^{17}$ A. M. Marshall, ${ }^{54}$ P. J. Marshall, ${ }^{60}$ G. Martelli, ${ }^{78}$ G. Martellotti, ${ }^{30}$ L. Martinazzoli, ${ }^{48, e}$ M. Martinelli, ${ }^{26, e}$ D. Martinez Santos, ${ }^{46}$ F. Martinez Vidal ${ }^{47}$ A. Massafferri, ${ }^{1}$ M. Materok, ${ }^{14}$ R. Matev, ${ }^{48}$ A. Mathad,${ }^{50}$ V. Matiunin, ${ }^{41}$ C. Matteuzzi, ${ }^{26}$ K. R. Mattioli, ${ }^{87}$ A. Mauri, ${ }^{32}$ E. Maurice, ${ }^{12}$ J. Mauricio, ${ }^{45}$ M. Mazurek, ${ }^{48}$ M. McCann, ${ }^{61}$ L. Mcconnell, ${ }^{18}$ T. H. Mcgrath, ${ }^{62}$ N. T. Mchugh, ${ }^{59}$ A. McNab, ${ }^{62}$ R. McNulty, ${ }^{18}$ J. V. Mead, ${ }^{60}$ B. Meadows, ${ }^{65}$ G. Meier, ${ }^{15}$ N. Meinert, ${ }^{76}$ D. Melnychuk, ${ }^{36}$ S. Meloni, ${ }^{26, e}$ M. Merk, ${ }^{32,80}$ A. Merli, ${ }^{25, p}$ L. Meyer Garcia, ${ }^{2}$ M. Mikhasenko, ${ }^{48}$ D. A. Milanes, ${ }^{74}$ E. Millard ${ }^{56}$ M. Milovanovic, ${ }^{48}$ M.-N. Minard, ${ }^{8}$ A. Minotti, ${ }^{26, \mathrm{e}}$ L. Minzoni, ${ }^{21, \mathrm{~d}}$ S. E. Mitchell, ${ }^{58}$ B. Mitreska, ${ }^{62}$ D. S. Mitzel, ${ }^{15}$ A. Mödden, ${ }^{15}$ R. A. Mohammed, ${ }^{63}$ R. D. Moise, ${ }^{61}$ S. Mokhnenko, ${ }^{82}$ T. Mombächer, ${ }^{46}$ I. A. Monroy, ${ }^{74}$ S. Monteil,${ }^{9}$ M. Morandin, ${ }^{28}$ G. Morello, ${ }^{23}$ M. J. Morello, ${ }^{29,0}$ J. Moron, ${ }^{34}$ A. B. Morris, ${ }^{75}$ A. G. Morris, ${ }^{56}$ R. Mountain, ${ }^{68}$ H. Mu, ${ }^{3}$ F. Muheim,,${ }^{58,48}$ M. Mulder, ${ }^{48}$ D. Müller, ${ }^{48}$ K. Müller, ${ }^{50}$ C. H. Murphy, ${ }^{63}$ D. Murray, ${ }^{62}$ P. Muzzetto, ${ }^{27,48}$ P. Naik, ${ }^{54}$ T. Nakada, ${ }^{49}$ R. Nandakumar, ${ }^{57}$ T. Nanut, ${ }^{49}$ I. Nasteva, ${ }^{2}$ M. Needham, ${ }^{58}$ I. Neri, ${ }^{21}$ N. Neri, ${ }^{25, p}$ S. Neubert, ${ }^{75}$ N. Neufeld, ${ }^{48}$ R. Newcombe,${ }^{61}$ E. M. Niel, ${ }^{11}$ S. Nieswand, ${ }^{14}$ N. Nikitin, ${ }^{40}$ N. S. Nolte,${ }^{64}$ C. Normand, ${ }^{8}$ C. Nunez, ${ }^{87}$ A. Oblakowska-Mucha, ${ }^{34}$ V. Obraztsov, ${ }^{44}$ T. Oeser,${ }^{14}$ D. P. O’Hanlon, ${ }^{54}$ S. Okamura, ${ }^{21}$ R. Oldeman,,${ }^{27, h}$ F. Oliva, ${ }^{58}$ M. E. Olivares, ${ }^{68}$ C. J. G. Onderwater, ${ }^{79}$ R. H. O’Neil, ${ }^{58}$ J. M. Otalora Goicochea, ${ }^{2}$ T. Ovsiannikova, ${ }^{41}$ P. Owen, ${ }^{50}$ A. Oyanguren, ${ }^{47}$ K. O. Padeken, ${ }^{75}$ B. Pagare,${ }^{56}$ P. R. Pais, ${ }^{48}$ T. Pajero, ${ }^{63}$ A. Palano, ${ }^{19}$ M. Palutan, ${ }^{23}$ Y. Pan, ${ }^{62}$ G. Panshin, ${ }^{84}$ A. Papanestis, ${ }^{57}$ M. Pappagallo, ${ }^{19, \mathrm{~g}}$ L. L. Pappalardo, ${ }^{21, \mathrm{~d}}$ C. Pappenheimer, ${ }^{65}$ W. Parker,${ }^{66}$ C. Parkes,${ }^{62}$ B. Passalacqua,${ }^{21}$ G. Passaleva, ${ }^{22}$ A. Pastore,${ }^{19}$ M. Patel, ${ }^{61}$ C. Patrignani, ${ }^{20, f}$ C. J. Pawley, ${ }^{80}$ A. Pearce, ${ }^{48}$ A. Pellegrino, ${ }^{32}$ M. Pepe Altarelli, ${ }^{48}$ S. Perazzini, ${ }^{20}$ D. Pereima,${ }^{41}$ A. Pereiro Castro, ${ }^{46}$ P. Perret,${ }^{9}$ M. Petric,${ }^{59,48}$ K. Petridis, ${ }^{54}$ A. Petrolini, ${ }^{24, b}$ A. Petrov, ${ }^{81}$ S. Petrucci, ${ }^{58}$ M. Petruzzo, ${ }^{25}$ T. T. H. Pham,${ }^{68}$ A. Philippov, ${ }^{42}$ L. Pica, ${ }^{29,0}$ M. Piccini, ${ }^{78}$ B. Pietrzyk, ${ }^{8}$ G. Pietrzyk, ${ }^{49}$ M. Pili, ${ }^{63}$ D. Pinci, ${ }^{30}$ F. Pisani, ${ }^{48}$ M. Pizzichemi, ${ }^{26,48, e}$ Resmi P. K., ${ }^{10}$ V. Placinta ${ }^{37}$ J. Plews,${ }^{53}$ M. Plo Casasus,${ }^{46}$ F. Polci, ${ }^{13}$ M. Poli Lener, ${ }^{23}$ M. Poliakova, ${ }^{68}$ A. Poluektov, ${ }^{10}$ N. Polukhina, ${ }^{83, s}$ I. Polyakov, ${ }^{68}$ E. Polycarpo, ${ }^{2}$ S. Ponce, ${ }^{48}$ D. Popov, ${ }^{6,48}$ S. Popov, ${ }^{42}$ S. Poslavskii, ${ }^{44}$ K. Prasanth,${ }^{35}$ L. Promberger, ${ }^{48}$

C. Prouve, ${ }^{46}$ V. Pugatch, ${ }^{52}$ V. Puill, ${ }^{11}$ H. Pullen, ${ }^{63}$ G. Punzi,${ }^{29, t}$ H. Qi ${ }^{3}$ W. Qian, ${ }^{6}$ J. Qin, ${ }^{6}$ N. Qin, ${ }^{3}$ R. Quagliani, ${ }^{49}$ B. Quintana, ${ }^{8}$ N. V. Raab, ${ }^{18}$ R. I. Rabadan Trejo, ${ }^{6}$ B. Rachwal, ${ }^{34}$ J. H. Rademacker, ${ }^{54}$ M. Rama ${ }^{29}$ M. Ramos Pernas, ${ }^{56}$ M. S. Rangel, ${ }^{2}$ F. Ratnikov, ${ }^{42,82}$ G. Raven, ${ }^{33}$ M. Reboud, ${ }^{8}$ F. Redi, ${ }^{49}$ F. Reiss, ${ }^{62}$ C. Remon Alepuz, ${ }^{47}$ Z. Ren, ${ }^{3}$ V. Renaudin, ${ }^{63}$ R. Ribatti, ${ }^{29}$ S. Ricciardi ${ }^{57}$ K. Rinnert, ${ }^{60}$ P. Robbe, ${ }^{11}$ G. Robertson, ${ }^{58}$ A. B. Rodrigues, ${ }^{49}$ E. Rodrigues, ${ }^{60}$ J. A. Rodriguez Lopez, ${ }^{74}$ E. R. R. Rodriguez Rodriguez, ${ }^{46}$ A. Rollings, ${ }^{63}$ P. Roloff, ${ }^{48}$ V. Romanovskiy, ${ }^{44}$ M. Romero Lamas, ${ }^{46}$ A. Romero Vidal, ${ }^{46}$ J. D. Roth, ${ }^{87}$ M. Rotondo, ${ }^{23}$ M. S. Rudolph,${ }^{68}$ T. Ruf, ${ }^{48}$ R. A. Ruiz Fernandez, ${ }^{46}$ J. Ruiz Vidal, ${ }^{47}$ A. Ryzhikov, ${ }^{82}$ J. Ryzka, ${ }^{34}$ J. J. Saborido Silva, ${ }^{46}$ N. Sagidova ${ }^{38}$ N. Sahoo, ${ }^{56}$ B. Saitta, ${ }^{27, h}$ M. Salomoni, ${ }^{48}$ 
C. Sanchez Gras, ${ }^{32}$ R. Santacesaria, ${ }^{30}$ C. Santamarina Rios, ${ }^{46}$ M. Santimaria, ${ }^{23}$ E. Santovetti, ${ }^{31, j}$ D. Saranin, ${ }^{83}$ G. Sarpis, ${ }^{14}$ M. Sarpis, ${ }^{75}$ A. Sarti, ${ }^{30}$ C. Satriano, ${ }^{30, u}$ A. Satta, ${ }^{31}$ M. Saur, ${ }^{15}$ D. Savrina, ${ }^{41,40}$ H. Sazak,${ }^{9}$ L. G. Scantlebury Smead,${ }^{63}$ A. Scarabotto, ${ }^{13}$ S. Schael, ${ }^{14}$ S. Scherl,${ }^{60}$ M. Schiller, ${ }^{59}$ H. Schindler, ${ }^{48}$ M. Schmelling, ${ }^{16}$ B. Schmidt, ${ }^{48}$ S. Schmitt, ${ }^{14}$ O. Schneider, ${ }^{49}$ A. Schopper, ${ }^{48}$ M. Schubiger, ${ }^{32}$ S. Schulte, ${ }^{49}$ M. H. Schune, ${ }^{11}$ R. Schwemmer, ${ }^{48}$ B. Sciascia, ${ }^{23,48}$ S. Sellam, ${ }^{46}$ A. Semennikov, ${ }^{41}$ M. Senghi Soares,${ }^{33}$ A. Sergi, ${ }^{24, b}$ N. Serra, ${ }^{50}$ L. Sestini, ${ }^{28}$ A. Seuthe, ${ }^{15}$ Y. Shang, ${ }^{5}$ D. M. Shangase ${ }^{87}$ M. Shapkin, ${ }^{44}$ I. Shchemerov ${ }^{83}$ L. Shchutska, ${ }^{49}$ T. Shears,${ }^{60}$ L. Shekhtman, ${ }^{43, i}$ Z. Shen, ${ }^{5}$ V. Shevchenko, ${ }^{81}$ E. B. Shields, ${ }^{26, e}$ Y. Shimizu, ${ }^{11}$ E. Shmanin,${ }^{83}$ J. D. Shupperd ${ }^{68}$ B. G. Siddi, ${ }^{21}$ R. Silva Coutinho, ${ }^{50}$ G. Simi ${ }^{28}$ S. Simone, ${ }^{19, \mathrm{~g}}$ N. Skidmore, ${ }^{62}$ T. Skwarnicki, ${ }^{68}$ M. W. Slater, ${ }^{53}$ I. Slazyk, ${ }^{21, d}$ J. C. Smallwood, ${ }^{63}$ J. G. Smeaton, ${ }^{55}$ A. Smetkina, ${ }^{41}$ E. Smith ${ }^{50}$ M. Smith, ${ }^{61}$ A. Snoch,${ }^{32}$ M. Soares, ${ }^{20}$ L. Soares Lavra, ${ }^{9}$ M. D. Sokoloff, ${ }^{65}$ F. J. P. Soler, ${ }^{59}$

A. Solovev, ${ }^{38}$ I. Solovyev, ${ }^{38}$ F. L. Souza De Almeida, ${ }^{2}$ B. Souza De Paula, ${ }^{2}$ B. Spaan,${ }^{15}$ E. Spadaro Norella, ${ }^{25, \mathrm{p}}$ P. Spradlin, ${ }^{59}$ F. Stagni, ${ }^{48}$ M. Stahl, ${ }^{65}$ S. Stahl, ${ }^{48}$ S. Stanislaus, ${ }^{63}$ O. Steinkamp, ${ }^{50,83}$ O. Stenyakin,${ }^{44}$ H. Stevens, ${ }^{15}$ S. Stone, ${ }^{68}$ M. Straticiuc, ${ }^{37}$ D. Strekalina ${ }^{83}$ F. Suljik, ${ }^{63}$ J. Sun, ${ }^{27}$ L. Sun, ${ }^{73}$ Y. Sun, ${ }^{66}$ P. Svihra, ${ }^{62}$ P. N. Swallow, ${ }^{53}$ K. Swientek ${ }^{34}$ A. Szabelski, ${ }^{36}$ T. Szumlak, ${ }^{34}$ M. Szymanski, ${ }^{48}$ S. Taneja ${ }^{62}$ A. R. Tanner, ${ }^{54}$ M. D. Tat ${ }^{63}$ A. Terentev, ${ }^{83}$ F. Teubert, ${ }^{48}$ E. Thomas, ${ }^{48}$ D. J. D. Thompson, ${ }^{53}$ K. A. Thomson,${ }^{60}$ V. Tisserand, ${ }^{9}$ S. T'Jampens, ${ }^{8}$ M. Tobin, ${ }^{4}$ L. Tomassetti, ${ }^{21, d}$ X. Tong, ${ }^{5}$ D. Torres Machado, ${ }^{1}$ D. Y. Tou, ${ }^{13}$ E. Trifonova, ${ }^{83}$ C. Trippl,${ }^{49}$ G. Tuci, ${ }^{6}$ A. Tully, ${ }^{49}$ N. Tuning, ${ }^{32,48}$ A. Ukleja, ${ }^{36}$ D. J. Unverzagt, ${ }^{17}$ E. Ursov, ${ }^{83}$ A. Usachov, ${ }^{32}$ A. Ustyuzhanin, ${ }^{42,82}$ U. Uwer, ${ }^{17}$ A. Vagner, ${ }^{84}$ V. Vagnoni, ${ }^{20}$ A. Valassi, ${ }^{48}$ G. Valenti, ${ }^{20}$ N. Valls Canudas, ${ }^{85}$ M. van Beuzekom, ${ }^{32}$ M. Van Dijk, ${ }^{49}$ H. Van Hecke, ${ }^{67}$ E. van Herwijnen, ${ }^{83}$ C. B. Van Hulse, ${ }^{18}$ M. van Veghel, ${ }^{79}$ R. Vazquez Gomez, ${ }^{45}$ P. Vazquez Regueiro, ${ }^{46}$ C. Vázquez Sierra, ${ }^{48}$ S. Vecchi, ${ }^{21}$ J. J. Velthuis, ${ }^{54}$ M. Veltri, ${ }^{22, v}$ A. Venkateswaran, ${ }^{68}$ M. Veronesi,${ }^{32}$ M. Vesterinen, ${ }^{56}$ D. Vieira, ${ }^{65}$ M. Vieites Diaz, ${ }^{49}$ H. Viemann ${ }^{76}$ X. Vilasis-Cardona ${ }^{85}$ E. Vilella Figueras, ${ }^{60}$ A. Villa, ${ }^{20}$ P. Vincent, ${ }^{13}$ F. C. Volle, ${ }^{11}$ D. Vom Bruch, ${ }^{10}$ A. Vorobyev, ${ }^{38}$ V. Vorobyev,${ }^{43, i}$ N. Voropaev, ${ }^{38}$ K. Vos, ${ }^{80}$ R. Waldi, ${ }^{17}$ J. Walsh, ${ }^{29}$ C. Wang, ${ }^{17}$ J. Wang, J. Wang, ${ }^{4}$ J. Wang, ${ }^{3}$ J. Wang, ${ }^{73}$ M. Wang, ${ }^{3}$ R. Wang, ${ }^{54}$ Y. Wang, ${ }^{7}$ Z. Wang, ${ }^{50}$ Z. Wang, ${ }^{3}$ Z. Wang, ${ }^{6}$ J. A. Ward, ${ }^{56}$ N. K. Watson, ${ }^{53}$ S. G. Weber, ${ }^{13}$ D. Websdale, ${ }^{61}$ C. Weisser, ${ }^{64}$ B. D. C. Westhenry,${ }^{54}$ D. J. White, ${ }^{62}$ M. Whitehead, ${ }^{54}$ A. R. Wiederhold, ${ }^{56}$ D. Wiedner, ${ }^{15}$ G. Wilkinson, ${ }^{63}$ M. Wilkinson, ${ }^{68}$ I. Williams, ${ }^{55}$ M. Williams,${ }^{64}$ M. R. J. Williams, ${ }^{58}$ F. F. Wilson, ${ }^{57}$ W. Wislicki, ${ }^{36}$ M. Witek, ${ }^{35}$ L. Witola, ${ }^{17}$ G. Wormser, ${ }^{11}$ S. A. Wotton, ${ }^{55}$ H. Wu ${ }^{68}$ K. Wyllie, ${ }^{48}$ Z. Xiang, ${ }^{6}$ D. Xiao, ${ }^{7}$ Y. Xie, ${ }^{7}$ A. Xu, ${ }^{5}$ J. Xu, ${ }^{6}$ L. Xu, ${ }^{3}$ M. Xu, ${ }^{7}$ Q. Xu, ${ }^{6}$ Z. Xu, ${ }^{5}$ Z. Xu, ${ }^{6}$ D. Yang, ${ }^{3}$ S. Yang, ${ }^{6}$ Y. Yang, ${ }^{6}$ Z. Yang, ${ }^{5}$ Z. Yang, ${ }^{66}$ Y. Yao, ${ }^{68}$ L. E. Yeomans, ${ }^{60}$ H. Yin, ${ }^{7}$ J. Yu ${ }^{71}$ X. Yuan, ${ }^{68}$ O. Yushchenko, ${ }^{44}$ E. Zaffaroni, ${ }^{49}$ M. Zavertyaev, ${ }^{16, s}$ M. Zdybal, ${ }^{35}$ O. Zenaiev, ${ }^{48}$ M. Zeng, ${ }^{3}$ D. Zhang, ${ }^{7}$ L. Zhang, ${ }^{3}$ S. Zhang, ${ }^{71}$ S. Zhang, ${ }^{5}$ Y. Zhang, ${ }^{5}$ Y. Zhang, ${ }^{63}$ A. Zharkova, ${ }^{83}$ A. Zhelezov, ${ }^{17}$ Y. Zheng, ${ }^{6}$ T. Zhou, ${ }^{5}$ X. Zhou, ${ }^{6}$ Y. Zhou, ${ }^{6}$ V. Zhovkovska, ${ }^{11}$ X. Zhu, ${ }^{3}$ X. Zhu, ${ }^{7}$ Z. Zhu, ${ }^{6}$ V. Zhukov, ${ }^{14,40}$ J. B. Zonneveld, ${ }^{58}$ Q. Zou, ${ }^{4}$ S. Zucchelli, ${ }^{20, f}$ D. Zuliani, ${ }^{28}$ and G. Zunica ${ }^{62}$

\title{
(LHCb Collaboration)
}

\author{
${ }^{1}$ Centro Brasileiro de Pesquisas Físicas (CBPF), Rio de Janeiro, Brazil \\ ${ }^{2}$ Universidade Federal do Rio de Janeiro (UFRJ), Rio de Janeiro, Brazil \\ ${ }^{3}$ Center for High Energy Physics, Tsinghua University, Beijing, China \\ ${ }^{4}$ Institute Of High Energy Physics (IHEP), Beijing, China \\ ${ }^{5}$ School of Physics State Key Laboratory of Nuclear Physics and Technology, Peking University, Beijing, China \\ ${ }^{6}$ University of Chinese Academy of Sciences, Beijing, China \\ ${ }^{7}$ Institute of Particle Physics, Central China Normal University, Wuhan, Hubei, China \\ ${ }^{8}$ Université Savoie Mont Blanc, CNRS, IN2P3-LAPP, Annecy, France \\ ${ }^{9}$ Université Clermont Auvergne, CNRS/IN2P3, LPC, Clermont-Ferrand, France \\ ${ }^{10}$ Aix Marseille Univ, CNRS/IN2P3, CPPM, Marseille, France \\ ${ }^{11}$ Université Paris-Saclay, CNRS/IN2P3, IJCLab, Orsay, France \\ ${ }^{12}$ Laboratoire Leprince-Ringuet, CNRS/IN2P3, Ecole Polytechnique, Institut Polytechnique de Paris, Palaiseau, France \\ ${ }^{13}$ LPNHE, Sorbonne Université, Paris Diderot Sorbonne Paris Cité, CNRS/IN2P3, Paris, France \\ ${ }^{14}$ I. Physikalisches Institut, RWTH Aachen University, Aachen, Germany \\ ${ }^{15}$ Fakultät Physik, Technische Universität Dortmund, Dortmund, Germany \\ ${ }^{16}$ Max-Planck-Institut für Kernphysik (MPIK), Heidelberg, Germany \\ ${ }^{17}$ Physikalisches Institut, Ruprecht-Karls-Universität Heidelberg, Heidelberg, Germany \\ ${ }^{18}$ School of Physics, University College Dublin, Dublin, Ireland \\ ${ }^{19}$ INFN Sezione di Bari, Bari, Italy
}




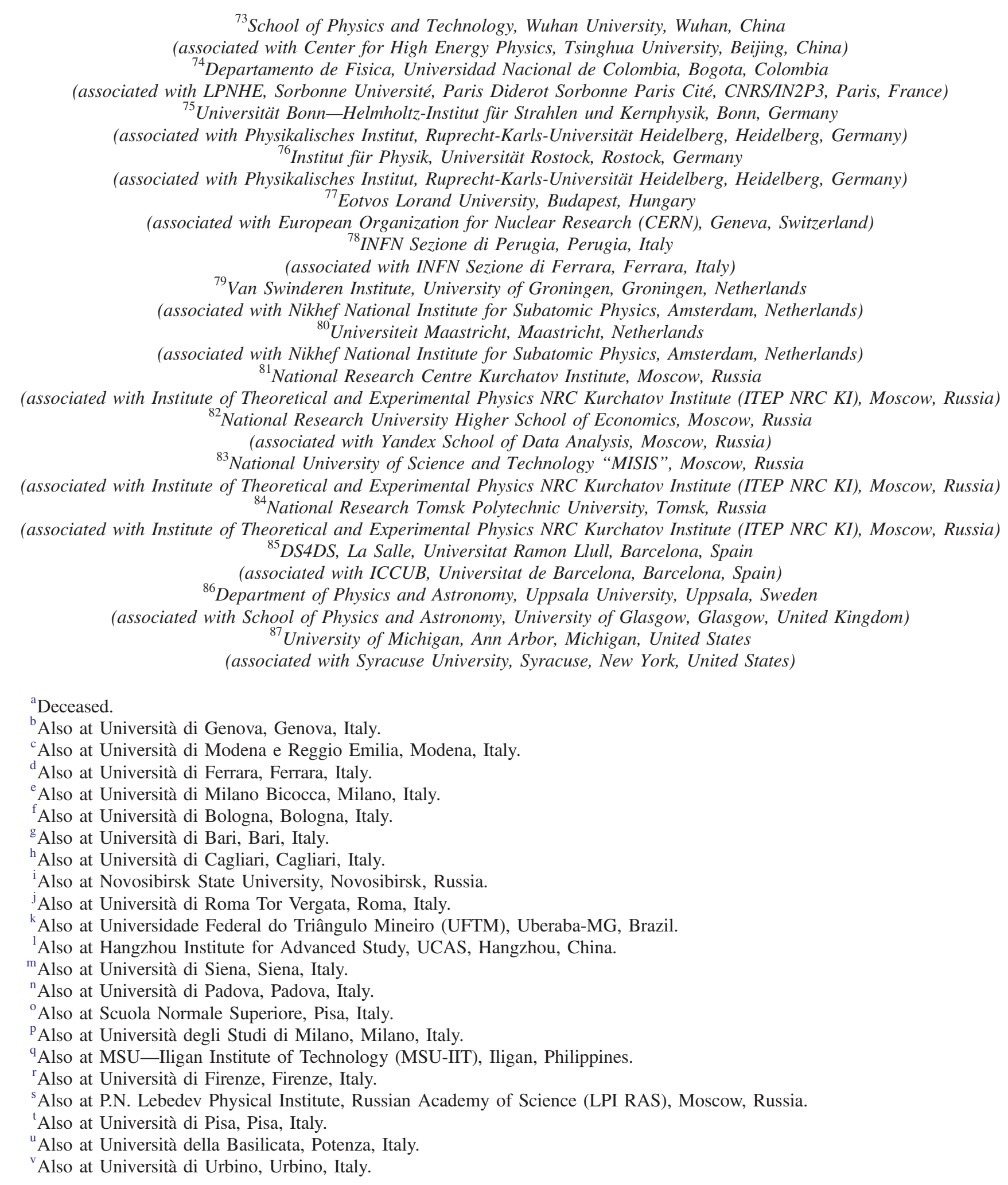

